\title{
Optimierte Produktionsplanung von Trommelgalvanikanlagen
}

\author{
S. Reimschüssel ${ }^{1}$, U. Fuchs ${ }^{2}$, G. Sand ${ }^{3}$
}

\section{Zusammenfassung}

Die Arbeit untersucht, inwieweit sich die zeitaufwendige und komplexe manuelle Erstellung von Trommelgalvanik-Fahrplänen mittels Methoden der gemischt-ganzzahligen Optimierung automatisieren lässt. Hierzu werden zwei reale Problemstellungen unterschiedlicher Größe und Struktur systematisch analysiert und auf das Optimierungsmodell von Steneberg abgebildet. Es wird gezeigt, dass die Eigenschaften realer Problemstellungen großteils abbildbar sind; Eigenschaften, welche dem Modell bislang noch fehlen, werden aufgedeckt und beschrieben. Bei beiden Problemstellungen stellt sich heraus, dass das Optimierungsmodell nicht innerhalb der erforderlichen Antwortzeit lösbar ist. Mit Hilfe der in dieser Arbeit entwickelten Modellvereinfachungsstrategie kann die Lösungszeit der Optimierungsmodelle deutlich reduziert werden. Obwohl bei Anwendung dieser Strategie Optimierungspotential verloren geht, ist es möglich, für das kleinere Anwendungsbeispiel innerhalb von 13 min Rechenzeit einen Fahrplan zu erstellen, welcher eine um 20 \% höhere Anlagenauslastung gegenüber der bisherigen manuellen Planung ermöglicht. Für kleinere Parametersätze des größeren Anwendungsbeispiels kann die Vereinfachungsstrategie ebenfalls erfolgreich angewendet und die Rechenzeitgrenzen des Modells aufgezeigt werden.

\section{Stichwörter}

Gemischt-ganzzahlige Optimierung, Automatisierung von Planungsprozessen, Fahrplanoptimierung, Trommelgalvanik

\section{Problemstellung}

In einer Trommelgalvanikanlage wird Schüttgut aus relativ unedlen Metallen mit dünnen Schichten edlerer Metalle überzogen. Eine derartige Anlage besitzt mehrere aneinandergereihte Bäder, welche mit unterschiedlichen, für die Beschichtung der Produkte notwendigen, Flüssigkeiten befüllt sind. Die mit Schüttgut befüllten Trommeln werden nacheinander in produktspezifischen Reihenfolgen in einen Teil der Bäder getaucht. Die Verweilzeiten in den Bädern dürfen innerhalb gegebener Spannen variieren. Der Transport der Trommeln zwischen den Bädern erfolgt automatisiert mittels Fahrwagen, die sich auf einer über den Bädern angebrachten Schiene bewegen. Mehrere Fahrwagen einer Galvanikanlage fahren auf derselben Schiene und können sich nicht überholen.

Das Fahrprogramm für die Fahrwagen bestimmt die Auslastung der Bäder und damit die Anlagenproduktivität. Dieses Programm basiert auf Fahrplänen, die momentan noch erfahrungsbasiert und händisch mit grafischer Unterstützung durch Microsoft Excel erstellt werden. Das folgende Bild 1 zeigt

1 Hochschule Pforzheim und Steinbeis-Transfer-Zentrum „Modellierung und Optimierung technischer Systeme“

2 UF automation

3 Hochschule Pforzheim und Steinbeis-Transfer-Zentrum „Modellierung und Optimierung technischer Systeme“ 
einen solchen, manuell erstellten Fahrplan für eine Trommelgalvanikanlage mit einer Linie, auf welcher sich 2 Fahrwagen bewegen. Auf der Abszissenachse ist die Zeit in Sekunden und auf der Ordinatenachse sind die 24 Positionen der Galvaniklinie aufgetragen. Die Anlage besitzt eine Wartungsstation (Position 1), eine Be-/Entladestation (Position 2) und 21 Prozessbäder (Positionen 4 bis 24). Zwischen der Be-/Entladestation und den restlichen Bädern der Anlage existiert ein Höhenunterschied, welcher durch einen Zwischenhub ausgeglichen wird (Position 3). Die Bäder 17 und 18, sowie 23 und 24 sind identische Bäder (befüllt mit derselben Flüssigkeit), von denen wahlweise eines zur Durchführung des Prozessschritts verwendet werden kann. Die Bäder 17 und 18 können dabei zeitgleich mit je einer Trommel belegt werden. Die Bäder 23 und 24 können hingegen nicht zeitgleich besetzt werden, da die Bäder alternierend gereinigt werden müssen. Die Anlage dient der Beschichtung zweier verschiedener Produkte, für deren jeweilige Bearbeitung beide Fahrwagen benötigt werden. Der erste Transportwagen übernimmt Transporte von Position 1 bis 13, der zweite Fahrwagen Transporte von Position 13 bis 24. Der Weg des ersten Fahrwagens zur Beschichtung des ersten Produkts ist in blau, der Weg des zweiten Fahrwagens zur Beschichtung des ersten Produkts in grün dargestellt. Soll das zweite Produkt beschichtet werden, so sind der rote beziehungsweise gelbe Alternativweg zu wählen.

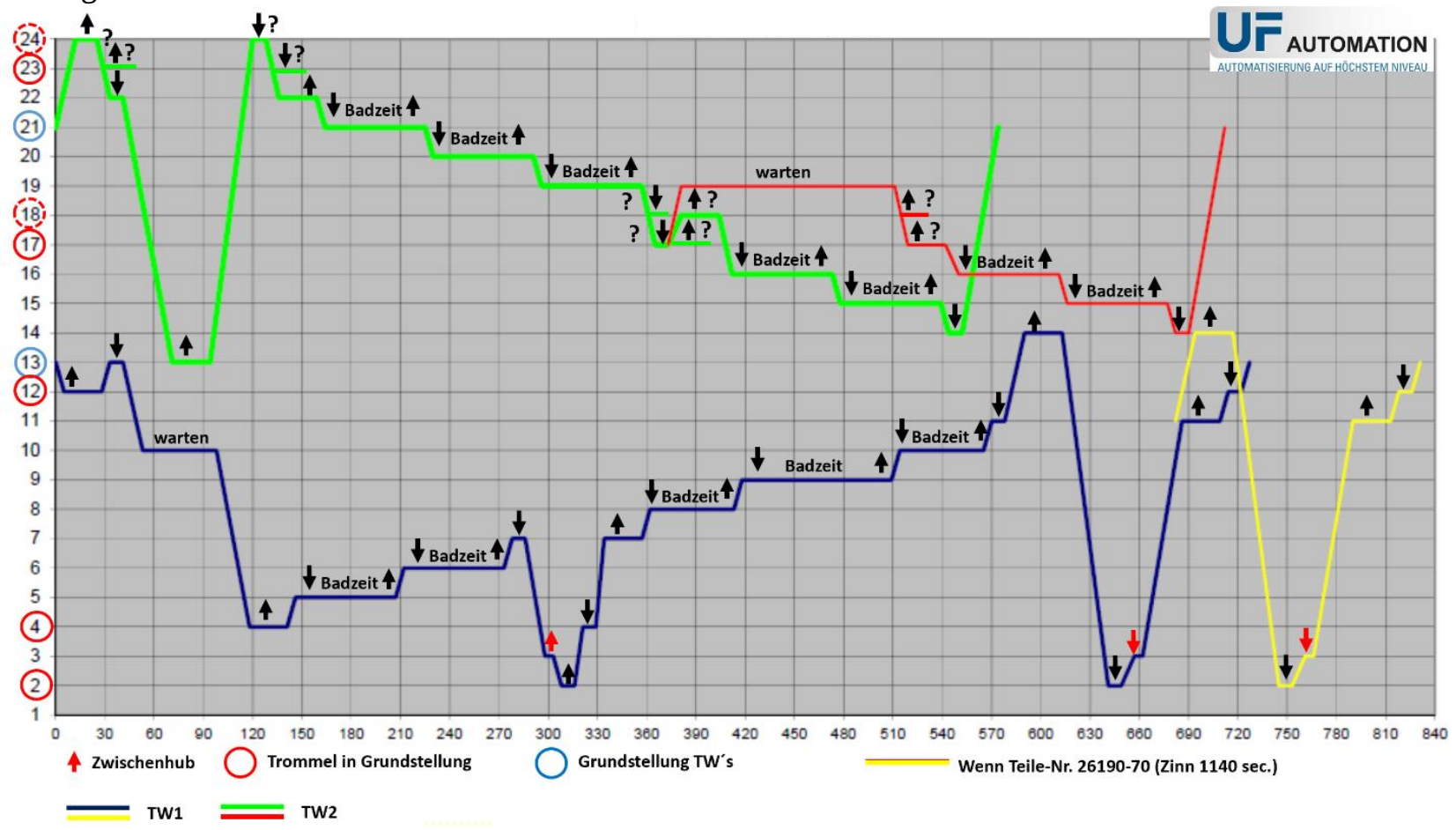

Bild 1: Manueller erstellter Fahrplan einer realen Trommelgalvanikanlage mit einer Linie auf welcher sich 2 Fahrwagen bewegen

Der manuell erstellte Fahrplan ist, zur Vereinfachung der Fahrplanerstellung, zyklisch und stark auf die Fahrwagen fokussiert. Das bedeutet, der Fahrplan wird mehrmals hintereinander ausgeführt, wobei die Fahrwagen in jedem Zyklus denselben Weg zurücklegen. Die Wege der Trommeln und die Prozessschritte hingegen sind nicht eingezeichnet und es sind mehrere Zyklen notwendig um den kompletten Weg einer Trommel zusammenhängend darzustellen. Die blau umkreisten Positionen sind die Grundpositionen (Positionen zu Beginn des Zyklus) der beiden Fahrwagen; die rot umkreisten Positionen sind die Grundpositionen der Trommeln. Die Trommeln selbst können nicht aus dem Prozess genommen werden und rotieren in der Anlage. Sie werden in der Be-/Entladestation befüllt, anschließend durch die Anlage gefahren und nach Beendigung aller Prozessschritte in der Be-/Entladestation wieder entleert und neu befüllt. Der menschliche Planer kalkuliert für diese Anlage fünf Trommeln ein. Zu Beginn eines Zyklus befinden sich Trommeln an den Positionen 2, 4 und 12, an Position 17 oder 18 und an Position 23 oder 24. Die Positionen der Trommeln in den identischen Bädern wechseln 
zwischen jedem Zyklus und sind im Fahrplan nicht explizit festgelegt. Während sich beispielsweise zu Beginn des ersten Durchlaufs eine Trommel an Position 17 befindet, ist zu Beginn des zweiten Durchlaufs das Bad 18 mit einer Trommel belegt. Während sich die Fahrwagen nach einem Zyklus wieder an exakt derselben Position befinden, verändern sich die Positionen der Trommeln mit jedem Zyklus. Zwar sind dieselben Positionen mit Trommeln besetzt, an den Positionen liegen jedoch andere Trommeln als zu Beginn des Zyklus. Beispielsweise befindet sich die Trommel, welche zu Beginn eines Zyklus an Position 4 lag, am Ende des Zyklus an Position 12.

Neben der vorgestellten einlinigen Anlage wurde zudem eine Großanlage untersucht, in der 30 verschiedene Produkte bearbeitet werden können und deren Bäder sich wie in Bild 2 gezeigt auf zwei parallele Linien verteilen. Zur ersten Linie gehören die Beladestation und die Bäder 1 bis 33 . Die zweite Linie umfasst die Bäder 34 bis 62, sowie die Entladestation. Beiden Linien können mehrere Fahrwagen zugeordnet werden, deren Fahrbereich auf eine Linie begrenzt ist. Am vorderen und hinteren Ende der Linien befinden sich Querumsetzer, welche die Trommeln zwischen den Linien transportieren. Der vordere Umsetzer setzt Trommeln von Linie 2 auf Linie 1 und der hintere Umsetzer Trommeln von Linie 1 auf Linie 2 um. Somit besitzt die Anlage keine lineare, sondern eine ringförmige Struktur. Die Be- und Entladestation der Großanlage befinden sich an zwei verschiedenen Positionen. Die Trommeln laufen im Kreis durch die Anlage und besitzen ausschließlich eine Bewegungsrichtung.

\begin{tabular}{|c|c|c|c|}
\hline \multirow{6}{*}{ 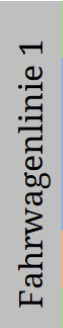 } & \multicolumn{2}{|c|}{ hinterer Umsetzer } & \\
\hline & Bad 33 & Bad 34 & $\ddot{\Xi}$ \\
\hline & $\cdots$ & $\ldots$ & 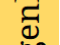 \\
\hline & Bad 1 & Bad 62 & 3 \\
\hline & Beladen & Entladen & $\vec{\tau}$ \\
\hline & \multicolumn{2}{|c|}{ jorderer Umsetzer } & \\
\hline
\end{tabular}

Bild 2: Ringförmige Struktur der Großanlage mit zwei parallelen Linien. Der Fahrbereich eines Fahrwagens beschränkt sich auf eine Linie.

Die Anlage ist noch im Aufbau, wodurch die Anzahl an Fahrwagen noch nicht festgelegt ist und damit einen weiteren Freiheitsgrad der Optimierung darstellt. Die manuelle Erstellung der Fahrpläne unterscheidet sich methodisch nicht von der einlinigen Anlage.

Die Planung der Fahrwege ist ein Optimierungsproblem, bei dem in begrenzter Zeit eine Vielzahl komplex wechselwirkender Entscheidungen getroffen werden muss. Das Ziel ist es, die Entscheidungen wie die Anzahl an Trommeln im Prozess oder welcher Fahrwagen welche Trommel zu welchem Zeitpunkt aus einem Bad hebt und senkt - derart zu treffen, dass die Zykluszeit minimal wird.

Hierfür soll ein gemischt-ganzzahliges lineares Optimierungsmodell (engl. Mixed Integer Linear Program) entwickelt werden, welches alle relevanten Problemeigenschaften abbildet und gleichzeitig in der verfügbaren Antwortzeit lösbar ist. Auf Basis der rechnerbasierten Optimierungsmethoden kann die manuelle Planung automatisiert erfolgen.

\section{Lösungsansatz}

Die Planungsaufgabe wird in einem MILP durch kontinuierliche und diskrete Variablen beschrieben, deren Wertebereich durch lineare, algebraische Gleichungen und Ungleichungen (Nebenbedingungen) beschränkt wird. Es verbleibt eine Vielzahl zulässiger Lösungen, die alle Nebenbedingungen einhalten und mit einer Zielfunktion bewertet werden. Gesucht ist die beste Lösung, welche alle Nebenbedingungen einhält. Allgemein kann ein mathematisches Optimierungsmodell wie folgt dargestellt werden, vergleiche [1]: 


$$
\min _{x, y}\left\{f(x, y) \mid \begin{array}{ll}
h x, y=0 \\
g(x, y) \geq 0
\end{array}, \begin{array}{l}
x \in X \subseteq \mathbb{R}^{n_{c}} \\
y \in Y \subseteq \mathbb{Z}^{n_{d}}
\end{array}\right\}
$$

Die Zielfunktion $f(x, y)$ soll, unter der Bedingung, dass die Vektoren der Gleichungsbedingungen $h x, y$ und der Ungleichungsbedingungen $g(x, y)$ erfüllt sind, minimiert werden. Der Vektor $x$ ist ein Element der Vektormenge $X$, welche eine Teilmenge des $n_{c}$ dimensionalen Vektorraums der reellen Zahlen darstellt. Der Vektor $y$ ist ein Element der Vektormenge $Y$, welche eine Teilmenge des $n_{d}$ dimensionalen Vektorraums der ganzen Zahlen darstellt. Dabei bezeichnet $n_{c}$ die Menge kontinuierlicher und $n_{d}$ die Menge ganzzahliger Variablen. Es handelt sich um ein MILP, wenn $n_{c}>0$ und $n_{d}>0$ ist und die Funktionen $f x, y, h x, y$ und $g(x, y)$ ausschließlich lineare Komponenten enthalten. Zur Implementierung mathematischer Optimierungsmodelle existieren verschiedene algebraische Modellierungssprachen und -umgebungen wie beispielsweise GAMS ${ }^{4}$, AMPL ${ }^{5}$ und OPL ${ }^{6}$. Für die Lösung der Optimierungsmodelle existieren Standardlöser wie beispielsweise CPLEX ${ }^{7}$, GUROBI $^{8}$ oder XPRESS 9 .

In Vorarbeiten konnte gezeigt werden, dass „einfache“ Job-Shop Modelle [2], wie das Flow-Shop oder Hybride Flow-Shop Modell zur Optimierung von Galvanikstraßen wie den hier betrachteten nicht ausreichen. Diesen Modellen fehlt es insbesondere an der Abbildbarkeit der Fahrwagen. Weiterhin wurde in den Vorarbeiten der aktuelle Stand der Forschung zur MILP-basierten Feinplanung von Trommelgalvanikanlagen mit Fahrwagen untersucht. Dabei konnten einige Forschungsarbeiten mit unterschiedlichen Modellformulierungen (beispielsweise [3], [4], [5], [6], [7]) gefunden werden, welche gegenüber den Job-Shop Modellen die Trommelgalvanik besser abbilden. Ein Vergleich der Modelle zeigt, dass das von Steneberg 2013 aufgestellte Optimierungsmodell [3] dem Anwendungsspektrum von UF automation am nächsten kommt. Dieses Modell wurde in der Modellierungsumgebung GAMS implementiert und mit Hilfe des Standardlösers CPLEX gelöst.

Das Steneberg-Modell besitzt die in Tabelle 1 aufgeführten Indexmengen, die in Tabelle 2 aufgeführten Parameter, die in Tabelle 3 aufgeführten Variablen und 12 weitere Blöcke von Hilfsvariablen.

Tabelle 1: Indexmengen des Steneberg-Modells

\begin{tabular}{|c|c|c|c|}
\hline 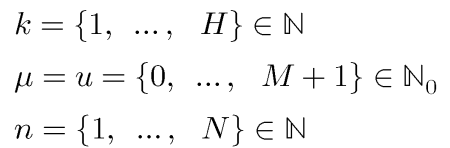 & $\begin{array}{l}\text { Fahrwagen } \\
\text { Prozessbäder } \\
\text { Bearbeitungsschemata }\end{array}$ & $\begin{array}{ll}a=\{1, & \ldots, \quad A\} \in \mathbb{N} \\
i=\{0, & \ldots, \quad I\} \in \mathbb{N}_{0}\end{array}$ & $\begin{array}{l}\text { Chargen } \\
\text { Prozessschritte }\end{array}$ \\
\hline
\end{tabular}

Jedem Bearbeitungsschema (Abfolge von Bädern zur Beschichtung eines Produkts) wird eine Anzahl an Chargen und eine Anzahl an Prozessschritten zugeordnet, sowie jedem Prozessschritt eines Bearbeitungsschemas ein konkretes Prozessbad. Der Abstand zwischen Prozessbädern, sowie der Mindestabstand zwischen Fahrwagen wird in Sekunden, die ein Fahrwagen braucht, um die entsprechende Strecke zurückzulegen, angegeben.

Tabelle 2: Parameter des Steneberg-Modells

\begin{tabular}{|c|c|c|c|}
\hline$C_{n} \in \mathbb{N}$ & Anzahl der Chargen & $T_{\mu}^{P} \in \mathbb{R}^{+}$ & Zeit zum Heben einer Trommel \\
\hline$P_{n} \in \mathbb{N}$ & Anzahl der Prozessschritte & $T_{\mu}^{L} \in \mathbb{R}^{+}$ & Zeit zum Senken einer Trommel \\
\hline
\end{tabular}

\footnotetext{
${ }^{4}$ https://www.gams.com/

${ }^{5}$ https://ampl.com/

${ }^{6}$ https://www.ibm.com/de-de/products/ilog-cplex-optimization-studio

${ }^{7}$ https://www.ibm.com/de-de/analytics/cplex-optimizer

${ }^{8}$ https://www.gurobi.com/

${ }^{9}$ https://www.fico.com/en/products/fico-xpress-solver
} 


\begin{tabular}{llll}
\hline$C_{n} \in \mathbb{N}$ & Anzahl der Chargen & $T_{\mu}^{P} \in \mathbb{R}^{+}$ & Zeit zum Heben einer Trommel \\
$v_{n, i} \in \mathbb{N}_{0}$ & Zuordnung eines Prozessbads & $P_{n, i}^{m i n} \in \mathbb{R}^{+}$ & Untere Grenze der Verweilzeit \\
$E_{\mu, u} \in \mathbb{R}^{+}$ & Abstand zweier Prozessbäder & $P_{n, i}^{m a x} \in \mathbb{R}^{+}$ & Obere Grenze der Verweilzeit \\
$\delta \in \mathbb{R}^{+}$ & Mindestabstand & $M$ & Big M Faktor \\
\hline
\end{tabular}

Ziel des Optimierungsmodells ist es, für jeden Prozessschritt $i$ einer Charge $a$ des Bearbeitungsschemas $n$ den Start- und Endzeitpunkt so zu wählen, dass die Zyklusdauer minimal wird. Dabei erhalten gleiche Prozessschritte unterschiedlicher Chargen eines Bearbeitungsschemas dieselbe Verweildauer im Prozessbad.

Tabelle 3: Variablen des Steneberg-Modells. Für die nicht aufgeführten 12 Blöcke von Hilfsvariablen siehe [3]

\begin{tabular}{|c|c|c|c|}
\hline$\Pi \in \mathbb{R}^{+}$ & Zyklusdauer & $t_{n, a, i}^{s} \in \mathbb{R}^{+}$ & Startzeitpunkt eines Prozessschritts \\
\hline$z_{n, a, i, k} \in\{0 ; 1\}$ & Fahrwagenzuordnung & $t_{n, a, i}^{e} \in \mathbb{R}^{+}$ & Endzeitpunkt eines Prozessschritts \\
\hline
\end{tabular}

Im Steneberg-Modell sind die Trommeln, welche in der Anlage rotieren, und deren Grundpositionen nicht explizit abgebildet. Durch das Festlegen der Start- und Endzeitpunkte und der Fahrwagenzuordnung ergeben sich die Anzahl und die Grundpositionen jedoch aus der Lösung des Modells. Das Modell besitzt 215 Blöcke von Randbedingungen zur Vermeidung von Kollisionen und zur Erreichbarkeit der Trommeln zu den festzulegenden Start- und Endzeitpunkten durch die Fahrwagen. Die restlichen 30 Blöcke von Randbedingungen definieren die Zielfunktion und sichern unter anderem die Einhaltung der Prozessschrittsequenz, der Verweilzeitgrenzen und der Bäderkapazitäten.

\section{Abbildbarkeit realer Anlagen mit dem Steneberg-Modell}

Das Steneberg-Modell wurde auf die in Abschnitt 1 beschriebene einlinige Trommelgalvanikanlage, sowie auf die Großanlage angewendet. Dabei konnte eine fehlerhafte Randbedingung im StenebergModell aufgedeckt werden, welche den Mindestabstand zwischen zwei Fahrwagen während eines Hebe- und eines Senkvorgang gewährleistet. Die entsprechende Konstellation ist in folgendem Bild 3 blau markiert. Hieraus lässt sich erkennen, dass der Abstand zwischen den Bädern $\mu_{n, i+1}$ und $\mu_{o, j}$ zum Abgleich mit dem Mindestabstand herangezogen werden muss.

\begin{tabular}{|c|c|c|c|}
\hline$\underbrace{\text { position }}_{t}$ & $\gamma=0, \tau=1$ & $\gamma=0, \tau=0$ & $\gamma=1$ \\
\hline \begin{tabular}{|l} 
pick up \\
- \\
pick up \\
(PP)
\end{tabular} & 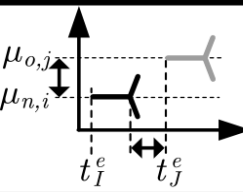 & 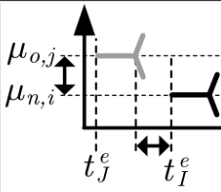 & $\hat{\mu_{o, j}} \mathbf{A}$ \\
\hline $\begin{array}{c}\text { lower } \\
- \\
\text { lower } \\
(\mathrm{LL})\end{array}$ & $t_{I+1}^{s} t$ & $t_{J+1}^{s \leftrightarrow} t$ & $\mu_{o, j+1} \hat{\mathbf{f}}$ \\
\hline \begin{tabular}{|c} 
lower \\
- \\
pick up \\
$(\mathrm{LP})$
\end{tabular} & 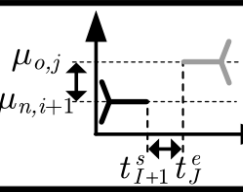 & 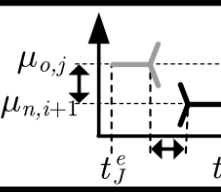 & $\begin{array}{c}\mu_{o, j} \\
\mu_{n, i+1}\end{array}$ \\
\hline $\begin{array}{c}\text { pick up } \\
- \\
\text { lower } \\
(\mathrm{PL})\end{array}$ & $\mu_{o, j+1} \mu_{n, i}$ & $\mu_{o, j+1}$ & $\mu_{o, j+1, \gamma} \hat{t}$ \\
\hline
\end{tabular}

Bild 3: Mögliche Konstellationen der Hebe- und Senkvorgänge. Das blau umrandete Szenario stellt den durch die fehlerhafte Randbedingung repräsentierten Fall sich zeitlich überlappender Hebe- und Senkvorgänge dar [3] 
Die Randbedingung (2) wurde durch die Randbedingung (3) mit korrigierten Indexmengen der Fahrzeit $E$ ersetzt.

$$
\begin{gathered}
\sum_{l \in \Gamma}\left(|k-l| \cdot \delta \cdot z_{J, l}\right) \leq E_{\mu_{n, i}, \mu_{o, j}}+M\left[2-\gamma_{I, J}^{L P}-z_{I, k}\right] \\
\sum_{l \in \Gamma}\left(|k-l| \cdot \delta \cdot z_{J, l}\right) \leq E_{\mu_{n, i+1}, \mu_{o, j}}+M\left[2-\gamma_{I, J}^{L P}-z_{I, k}\right]
\end{gathered}
$$

Aufgrund der Komplexität des Steneberg-Modells mit seinen 245 Blöcken von Randbedingungen wurde zunächst untersucht, welche Aspekte der realen Problemstellungen ohne Abändern des Modells durch geeignetes Anpassen der Parametersätze abgebildet werden können.

\subsection{Einlinige Anlage}

Die Nummerierung der Prozessbäder, sowie der Be-/Entlade- und der Wartungsstation kann wie in Tabelle 4 gezeigt folgendermaßen angepasst werden:

- Die Wartungsstation (Position 1) wird in die manuelle Planung nicht einbezogen und kann im Optimierungsmodell ebenfalls vernachlässigt werden.

- Zur Abbildung des Zwischenhubs wurde im Gegensatz zur manuellen Planung keine zusätzliche Position vergeben, sondern die Dauer des Zwischenhubs zur Fahrzeit zwischen der Be-/Entladestation und den anderen Positionen hinzuaddiert.

- Die identischen Bäder 23 und 24, welche nicht zeitgleich besetzt werden können, sind durch ein durchgängig besetzbares Bad ohne Verlust an Optimierungspotential abbildbar.

Tabelle 4: Anpassung der Positionen aus der manuellen Planung für das Steneberg-Modell

\begin{tabular}{l|c|c|c|c|c|c|c|c|c|c|c|c|c|c|c|c|c|c|c|c|c|c|c|c}
\hline Bezeichnung & W & B/E & Z & P & P & P & P & P & P & P & P & P & P & P & P & P & P & P & P & P & P & P & P & P \\
\hline $\begin{array}{l}\text { Position in der } \\
\text { manuellen Planung }\end{array}$ & 1 & 2 & 3 & 4 & 5 & 6 & 7 & 8 & 9 & 10 & 11 & 12 & 13 & 14 & 15 & 16 & 17 & 18 & 19 & 20 & 21 & 22 & 23 & 24 \\
\hline $\begin{array}{l}\text { Position im } \\
\text { Optimierungsmodell }\end{array}$ & - & 0 & - & 1 & 2 & 3 & 4 & 5 & 6 & 7 & 8 & 9 & 10 & 11 & 12 & 13 & 14 & 15 & 16 & 17 & 18 & 19 & 20 & - \\
\hline
\end{tabular}

$\mathrm{W}=$ Wartungsstation $\quad \mathrm{B} / \mathrm{E}=\mathrm{Be}-/$ Entladestation $\quad \mathrm{Z}=$ Zwischenhub $\quad \mathrm{P}=$ Prozessbad

Im Optimierungsmodell ist die Entnahme der ersten Trommel aus der Be-/Entladestation zum Zeitpunkt 0 erforderlich. Zum besseren Vergleich des manuellen Plans mit dem optimierten Plan ist es hilfreich, die Zeitachse des manuellen Plans so zu verschieben, dass auch im manuellen Plan die erste Trommel zum Zeitpunkt 0 entnommen wird. Aufgrund der zyklischen Planung ändert sich hierdurch nichts an der Planung, sondern lediglich an der Darstellung.

Die Abtropfzeiten werden zu den Hebezeiten hinzuaddiert. Wie in der manuellen Planung wird für die Abtropfzeiten ein fester Wert angenommen. Dies stellt eine Vereinfachung dar, da die Abtropfzeiten in der Realität innerhalb bestimmter Spannen variieren dürfen.

Auch die feste Vorgabe der Grenzen für die Verweilzeiten der Trommeln in den Bädern sind eine Vereinfachung der Realität. In seltenen Fällen dürfen die Grenzen zu Gunsten einer besseren Planung überschritten werden. Diese Möglichkeit wird bei der manuellen Planung ausgenutzt. Um einen aussagekräftigen Vergleich des manuellen Plans mit dem optimierten Plan anzustellen, werden die vom menschlichen Planer ausgenutzten Grenzen im Modell ebenfalls gelockert.

Die Fahrzeiten zwischen den Bädern sind für 7 Strecken exakt gemessen. Der Abstand der Bäder zueinander ist konstant. Die Abhängigkeit der Fahrzeiten vom Abstand der Bäder kann durch lineare Interpolation und Extrapolation der Messpunkte approximiert werden. Die Fahrzeiten sind von der 
Beladung der Trommel abhängig; dieser Aspekt ist im Modell noch nicht abbildbar. In der manuellen Planung wird zum Abfangen dieser Ungenauigkeiten am Zyklusende ein zeitlicher Puffer eingeplant. Der erste Prozessschritt erhält im Steneberg-Modell nur einen End- und keinen Startzeitpunkt. Umgekehrt erhält der letzte Prozessschritt nur einen Start- und keinen Endzeitpunkt. Ein Vergleich des Start- beziehungsweise Endzeitpunkts des ersten und letzten Prozessschritts, welche im vorliegenden Anwendungsfall dem Be- und Entladen entspricht, fehlt dem Modell. Folglich ist nicht kontrollierbar, wie lange sich eine Trommel in der Be-/Entladestation aufhält und wie viele Trommeln sich zeitgleich in der Anlage befinden.

Die identischen, zeitgleich besetzbaren Prozessbäder 14 und 15 (nach angepasster Nummerierung) sind im Steneberg-Modell nicht direkt abbildbar. Sie können dennoch eingebracht werden, indem für die Beschichtung eines Produkts zwei unterschiedliche Bearbeitungsschemata definiert werden. Die Schemata sind bis auf die Zuordnung zu einem identischen Bad identisch: Dem entsprechenden Prozessschritt wird in einem Bearbeitungsschema das Bad 14, im anderen Bearbeitungsschema das Bad 15 zugeordnet. Bei einer getrennten Optimierung der Aufträge führt diese Art der Parametrierung bei einer gleichmäßigen Aufteilung der Chargen auf die Bearbeitungsschemata zu keinem Verlust an Optimierungspotential.

\subsection{Großanlage mit zwei Linien}

Die beiden Linien der Großanlage sind wie in Abschnitt 1 beschrieben an den Enden mit jeweils einem Querumsetzer verbunden. Diese übernehmen den Transport der Trommeln von einer auf die andere Linie. Für die Umsetzdauer auf eine andere Linie existiert eine fest einzuhaltende Zeitspanne, sowie feste Senk-, Hebe- und Abtropfzeiten. Sie verhalten sich somit aus Sicht des Modells identisch zu den Prozessbädern und können als solche modelliert werden.

Das Steneberg-Modell kann ausschließlich linienförmige Anlagen abbilden, weshalb die in Bild 2 dargestellte Ringstruktur aufgebrochen werden muss. Dies erfolgt im Modell durch die Nummerierung der Beladestation, Entladestation, Prozessbäder und Umsetzer. Erhält die Beladestation die Position 0 und wird die Nummerierung im Uhrzeigersinn entlang der Ringstruktur fortgeführt, so teilt sich, wie Bild 4 a) zeigt, die Fahrwagenreihe 1 in zwei Bereiche auf. Die Nummerierung der Fahrwagen ist im Modell so zu wählen, dass der erste Fahrwagen Transporte von und zu der ersten Position übernimmt und der letzte Fahrwagen Transporte von und zu der letzten Position. Somit muss bei der Auftrennung an der Beladestation die Nummerierung der Fahrwagen wie in Bild 4 a) in rot dargestellt erfolgen. Für die Bestimmung des Fahrwagenabstands werden die Fahrwagennummern herangezogen. Mit der Nummerierung aus Bild 4 a) müssten Fahrwagen 1 und Fahrwagen 8 einen Mindestabstand von $7 \cdot \delta$ zueinander einhalten. In der Realität genügt jedoch ein Mindestabstand von $\delta$. Folglich ist nur eine korrekte Berechnung des Mindestabstands bei einer Auftrennung der Ringstruktur an einem der Querumsetzer wie in Bild 4 b) gezeigt möglich, da an diesen Stellen die Fahrwagenreihen enden.

a)

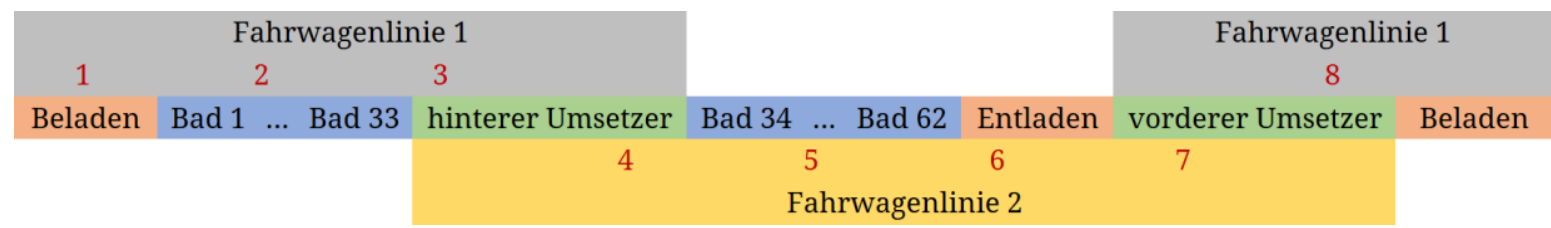

b)

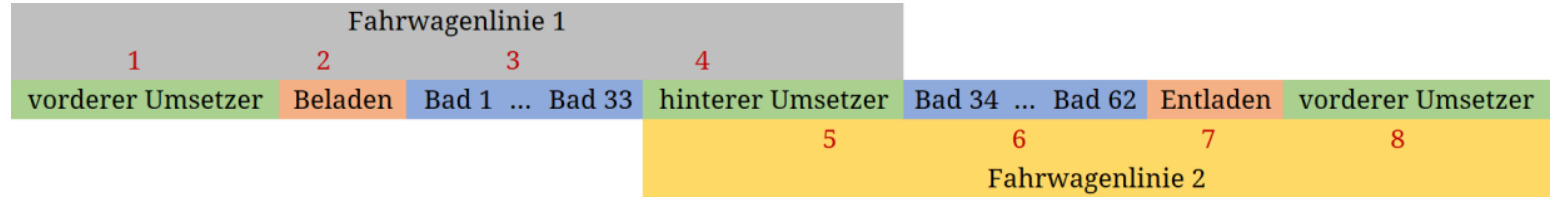

Bild 4: Auftrennen der Ringstruktur der Großanlage an unterschiedlichen Positionen. a) Auftrennen an der Beladestation, b) Auftrennen am vorderen Umsetzer; in rot dargestellt ist die Nummerierung der Fahrwagen 
Aus Sicht des Modells existiert nur eine Linie und somit nur die Begrenzung der Fahrwagen am Anfang und am Ende dieser Linie. Die reale Anlage besitzt jedoch zwei Linien und damit vier Begrenzungen der Fahrwagen. Durch die in Bild 4 b) dargestellte Auftrennung der Ringstruktur können die Fahrwagen über den hinteren Umsetzer hinaus weiterfahren und die mechanische Begrenzung der realen Anlage wird nicht eingehalten. Das Festsetzen der Variablenwerte für die Fahrwagenzuordnung, sodass Zuweisungen eines Fahrwagens der Linie 1 zu einem Transport auf Linie 2 und umgekehrt unterbunden wird, verhindert das Überschreiten der Grenze lediglich wenn der Fahrwagen eine Trommel transportiert. Eine Leerfahrt über den hinteren Umsetzer hinaus, wie beispielsweise eine Ausweichfahrt, kann hierdurch aber nicht verhindert werden. Wie bereits bei der einlinigen Anlage ist auch hier mit dem Steneberg-Modell die Anzahl der Trommeln in und deren Verweilzeit an der ersten und letzten Position nicht kontrollierbar. In diesem Fall handelt es sich um den vorderen Umsetzer. Die Fahrwagenanzahl wird im Modell durch einen festen Parameter abgebildet, ist in der Realität für diesen Anwendungsfall jedoch ein Freiheitsgrad.

\section{Lösen des Modells für reale Parametersätze}

Wird das Modell mit den Daten der Anlage parametriert, kann mit dem Standardlöser CPLEX innerhalb von $24 \mathrm{~h}$ keine zulässige Lösung gefunden werden. Der verwendete Rechner besitzt einen Intel ${ }^{\circledR}$ Core $^{\mathrm{TM}}$ i7-10510U Prozessor mit einer Grundtaktfrequenz von 1,8 GHz und insgesamt 8 Threads. Zur Lösung der Modelle wurde 1 Thread verwendet. Bei allen hier angegebenen Zeiten handelt es sich um „wall clock time“. Zur Reduktion der Lösungszeit wurden Vereinfachungsstrategien aus der manuellen Planung in das Modell zu übernommen. In der manuellen Planung werden für alle Aufträge individuelle Pläne erstellt. Diese werden je nach Auftragslage miteinander kombiniert. Die manuell erstellten Pläne sind zyklisch und jeder Prozessschritt des Auftrags wird, auch wenn mehrere Chargen bearbeitet werden sollen und sich mehrere Trommeln in der Anlage befinden, nur einmal eingeplant. Hierdurch ergibt sich gegenüber azyklischen Plänen und Plänen, in denen jeder Prozessschritt explizit geplant wird, ein sehr viel kürzerer Planungshorizont und die Planungskomplexität wird reduziert. Gleichzeitig geht hierdurch aber auch Optimierungspotenzial verloren.

Das Prinzip dieser Vereinfachung veranschaulicht der Fahrplan der sechsten Testinstanz aus der Veröffentlichung von Steneberg in Bild 5. In dieser Testinstanz werden zwei Bearbeitungsschemata mit je 2 Chargen eingeplant. Zu Bearbeitungsschema 1 gehören die hell- und dunkelorangene Charge, zu Bearbeitungsschema 2 die hell- und dunkelgrüne Charge. Ab dem Zeitpunkt $\frac{\Pi}{2}$ wiederholt sich der Plan, wobei die Chargen eines Bearbeitungsschemas vertauscht sind. Da beide Chargen eines Bearbeitungsschemas im linken Teil und im rechten Teil des Plans auf exakt dieselbe Weise bearbeitet werden, erstellt der menschliche Planer ausschließlich den linken Teil des Plans. Im Optimierungsmodell wird dies umgesetzt, indem 2 Bearbeitungsschemata mit je einer Charge definiert werden. Diese Vereinfachung führt immer dann zu keinem Verlust an Abbildungsgenauigkeit, wenn die Anzahl der Chargen jedes Bearbeitungsschemas um den gleichen Faktor reduziert wird. Sollen vom ersten Bearbeitungsschema beispielsweise 3 Chargen bearbeitet werden, während gleichzeitig vom zweiten Bearbeitungsschema 2 Chargen bearbeitet werden, ist keine Vereinfachung des Parametersatzes möglich.

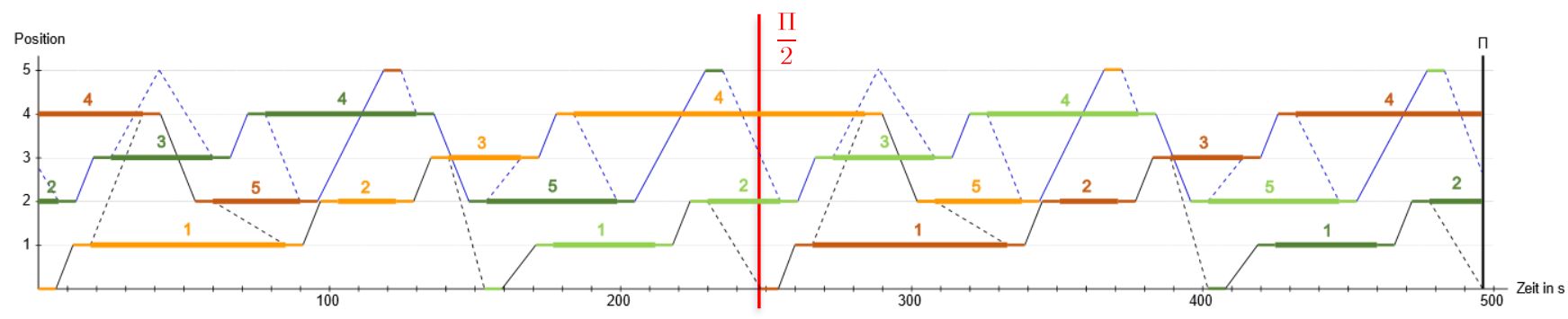

Bild 5: Ergebnis der sechsten Testinstanz von Steneberg mit 2 Bearbeitungsschemata orange und grün mit jeweils 2 Chargen hell und dunkel. Ab dem Zeitpunkt $\frac{\Pi}{2}$ wiederholt sich der Plan mit vertauschten Chargen. 
Um das Steneberg-Modell mit Standardlösern lösen zu können, wurden diese Heuristiken der manuellen Planung auf das Modell abgebildet. Die Anzahl der Chargen $a$ je Bearbeitungsschema $n$ wird auf 1 reduziert. Aufgrund des identischen Bads der einlinigen Anlage werden 2 Bearbeitungsschemata verwendet. Weiterhin beschränkt sich die Optimierung auf den ersten Auftrag. Hierdurch kann die Modellgröße derart reduziert werden, dass der CPLEX-Löser innerhalb von 13 min eine optimale Lösung findet. Um den optimierten Plan mit der manuellen Lösung vergleichen zu können wurde dieser, insbesondere mit Blick auf die Modellschwächen, untersucht. Dabei konnten keine Fehler, das heißt keine Abschnitte im Plan gefunden werden, welche in der Realität nicht durchführbar sind. Beispielsweise befindet sich zu jedem Zeitpunkt nur eine Trommel in der Be-/Entladestation. Zu beachten ist, dass die Fahrzeiten approximiert wurden und von den realen Zeiten abweichen können. Dieser Aspekt trifft jedoch sowohl auf das Optimierungsmodell als auch auf die manuelle Planung zu. Der in der manuellen Planung am Ende des Zyklus eingebrachte Puffer zum Abfangen dieser Abweichungen wurde zum Vergleich mit dem optimierten Plan herausgerechnet. Mit dem vom Optimierungsmodell erstellten Fahrplan lassen sich innerhalb von 17880 Sekunden 30 Chargen bearbeiten, während mit dem manuell erstellten Plan für dieselbe Anzahl an Chargen 22110 Sekunden benötigt werden. Dies entspricht einer Verbesserung um 19,13\%.

Die Optimierung zeigt, dass eine weitere Trommel im Prozess untergebracht werden kann, indem die identischen Bäder besser ausgelastet werden. In folgendem Bild 7 ist links ein Ausschnitt des manuell erstellten und rechts ein Ausschnitt des mit dem Optimierungsmodell erstellten Fahrplans dargestellt. Es ist zu sehen, dass sich der Fahrweg von Fahrwagen 2 (grüne Linie) im Bereich der identischen Bäder 17/14 und 18/15 in den Plänen unterscheidet. Im optimierten Fahrplan wird noch während des Prozessschritts 15 eine Trommel aus dem Bad 17 entnommen. Nach dem Prozessschritt 15 kann die Trommel direkt in das frei gewordene Bad gesetzt werden. Die Zeitspanne, in der Bad 17 nicht belegt ist wird hierdurch möglichst kurzgehalten.
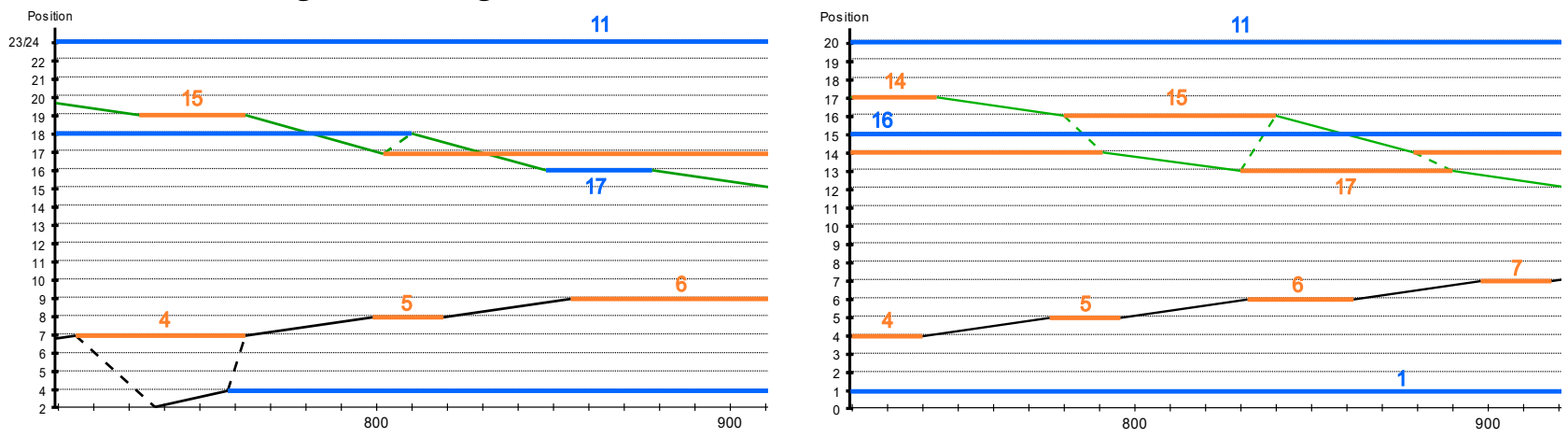

Bild 6: Vergleich des manuell erstellten Fahrplans (links) mit dem optimierten Fahrplan (rechts)

Diese Vereinfachung wurde ebenfalls auf den Parametersatz der Großanlage angewendet und das Modell exemplarisch für drei unterschiedliche Auftragsarten gelöst. Dabei zeigte sich, dass die Vereinfachung nicht genügt, um die Lösungszeit auf unter eine Stunde zu reduzieren. Die Ergebnisse können der folgenden Tabelle 5 entnommen werden.

Tabelle 5: Lösungszeiten bei Anwendung des Steneberg-Modells mit Vereinfachung auf die Großanlage

\begin{tabular}{ccccr}
\hline Auftragsart & $\begin{array}{c}\text { Anzahl der } \\
\text { Prozessschritte } \boldsymbol{i}\end{array}$ & $\begin{array}{c}\text { Anzahl der } \\
\text { Bearbeitungsschemata } \boldsymbol{n}\end{array}$ & $\begin{array}{c}\text { Anzahl der } \\
\text { Fahrwagen } \boldsymbol{h}\end{array}$ & $\begin{array}{r}\text { Lösungszeit in } \\
\text { [h:min:s] }\end{array}$ \\
\hline A & 25 & 2 & 2 & $00: 00: 05$ \\
A & 25 & 2 & 4 & $00: 08: 33$ \\
A & 25 & 2 & 6 & $00: 53: 17$ \\
A & 25 & 2 & 8 & $01: 34: 28$
\end{tabular}




\begin{tabular}{ccccr}
\hline Auftragsart & $\begin{array}{c}\text { Anzahl der } \\
\text { Prozessschritte } \boldsymbol{i}\end{array}$ & $\begin{array}{c}\text { Anzahl der } \\
\text { Bearbeitungsschemata } \boldsymbol{n}\end{array}$ & $\begin{array}{c}\text { Anzahl der } \\
\text { Fahrwagen } \boldsymbol{h}\end{array}$ & $\begin{array}{r}\text { Lösungszeit in } \\
\text { [h:min:s] }\end{array}$ \\
\hline B & 13 & 4 & 2 & $00: 00: 17$ \\
B & 13 & 4 & 4 & $00: 06: 11$ \\
B & 13 & 4 & 6 & $00: 14: 44$ \\
B & 13 & 4 & 8 & $00: 19: 58$ \\
C & 30 & 2 & 2 & $00: 00: 26$ \\
C & 30 & 2 & 3 & $00: 04: 55$ \\
C & 30 & 2 & 4 & $10: 41: 31$ \\
C & 30 & 2 & 5 & $>48: 00: 00$ \\
\hline
\end{tabular}

\section{Fazit und Ausblick}

Die Arbeit zeigt, dass das Steneberg-Modell viele Aspekte der Feinplanung realer Trommelgalvanikanlagen abbildet. Es konnten jedoch Modellschwächen identifiziert werden, die entweder zu schlechteren oder zu fehlerhaften Plänen führen. Im nächsten Schritt soll das Modell daher, um die fehlenden Aspekte erweitert werden. Weiterhin wird deutlich, dass die Übertragung manueller Heuristiken auf das Optimierungsmodell die Lösungszeit deutlich reduzieren kann. Die Anwendung des vereinfachten Modells auf die Großanlage zeigt jedoch, dass weitere Lösungsansätze entwickelt werden müssen, um Fahrpläne für größere Anlagen mit mehreren Linien, über 60 Bäder und mehr als 4 Fahrwagen zu berechnen. In Folgearbeiten sollen daher die Modellstruktur analysiert und geeignete Ansätze wie lazy constraints, Aggregation oder Dekomposition untersucht werden, um die Modellgröße weiter zu reduzieren und die Berechnung zu parallelisieren. Zudem soll die Belegungsplanung um die drei Planungsschritte Ressourcenplanung, Auftragsplanung und reaktive Planung erweitert werden, um die gesamten Prozesse zum Betrieb der Galvanikanlagen automatisiert zu planen und zu steuern, sowie auf unvorhergesehene Ereignisse zu reagieren.

\section{Literatur}

[1] J. Kallrath, Gemischt-ganzzahlige Optimierung: Modellierung in der Praxis, Wiesbaden: Springer Vieweg, Wiesbaden, 2013.

[2] W. Domschke, A. Scholl und S. Voß, Produktionsplanung - Ablauforganisatorische Aspekte, Berlin Heidelberg: Springer-Verlag , 1997.

[3] S. C. Steneberg, „MILP model for multi-product, multi-part and multi-hoist cycle shops,“ in IEEE International Conference on Industrial Technology (ICIT 2013), 2013.

[4] N. P. Basán und C. A. Méndez, „Hybrid MILP/Simulation/Heuristic Algorithms to Complex Hoist Scheduling Problems,“ Computer Aided Chemical Engineering, Bd. 38, 2016.

[5] Y. Jiang und J. Liu, „A new model and an efficient branch-and-bound solution for cyclic multi-hoist scheduling,“ IEE Transactions, Bd. 46, 2014.

[6] J. M. Y. Leung, G. Zhang, X. Yang, R. Mak und K. Lam, „Optimal Cyclic Multi-Hoist Scheduling: A Mixed Integer Programming Approach,“ Operations Research, Bd. 52, 2004.

[7] Z. Zhou und L. Li, „A solution for cyclic scheduling of multi-hoists without overlapping,“ Annals of Operations Research, Bd. 168, 2009. 